\title{
Violencia de Barrio y Crianza Materna en Medellín-Roma: Rol de la Autoeficacia Emocional Negativa
}

\section{Neighborhood Violence and Maternal Parenting in Medellin-Rome: The Role of Negative Emotional Self-Efficacy}

\author{
Luz Magnolia Tilano Vega ${ }^{1}$ Paula Luengo Kanacri², Concetta Pastorelli³, Laura Di Giunta ${ }^{3}$, \\ Carolina Lunetti ${ }^{3}$, Liliana Uribe Tirado ${ }^{1}$, Eriona Thartori ${ }^{3}$ y Jennifer Lansford ${ }^{4}$ \\ ${ }^{1}$ Facultad de Psicología, Universidad de San Buenaventura \\ ${ }^{2}$ Escuela de Psicología, Pontificia Universidad Católica de Chile \\ ${ }^{3}$ Dipartimento di Psicologia, Sapienza Università di Roma \\ ${ }_{4}^{4}$ Center for Child and Family Policy, Duke University
}

\begin{abstract}
El estudio analiza el efecto de la percepción de violencia de barrio en la crianza positiva y si este es mediado por la autoeficacia percibida para la gestión de emociones negativas de las madres comparando los países de Colombia e Italia. Los participantes fueron 434 madres e hijos de 8 a 10 años (108 díadas de Medellín-Colombia y 109 díadas de Roma-Italia), seleccionados por conveniencia, de forma no probabilística y proporcionalmente al estrato social de cada ciudad. Madres e hijos respondieron la Escala de Percepción de Violencia de Barrio. Las madres además respondieron la Escala de Autoeficacia Emocional y los hijos el Cuestionario de Aceptación-Rechazo/Control. Un análisis multigrupo mostró que el rol de la gestión de emociones negativas de las madres es diferente en ambos países. En Medellín-Colombia la autoeficacia percibida para la gestión de emociones negativas de las madres media el efecto de la percepción de violencia de barrio sobre la crianza positiva. En Italia, la autoeficacia de las madres con respecto al manejo de las emociones negativas no media esta interacción. La autoeficacia percibida para la gestión de emociones negativas de las madres colombianas explica indirectamente el efecto adverso de la percepción de violencia de barrio sobre las prácticas educativas parentales positivas. Con las madres de Roma-Italia, no se evidencia una relación significativa entre la autoeficacia percibida para la gestión de emociones negativas con la crianza positiva.
\end{abstract}

Palabras clave: crianza positiva, calidad de la relación madre-hijo, autoeficacia emocional, violencia de barrio

The study analyzes the effect of perceived neighborhood violence on positive parenting and whether it is mediated by mothers' perceived self-efficacy in managing negative emotions, comparing the situation in Colombia and Italy. Participants were 434 mothers and children aged 8 to 10 years (108 dyads from Medellin, Colombia and 109 dyads from Rome, Italy), selected by convenience sampling, non-probabilistically, and proportionally to the social strata in each city. Mothers and children answered the Neighborhood Violence Perception Scale. Mothers also answered the Emotional Self-Efficacy Scale and children took the Acceptance-Rejection/Control questionnaire. A multigroup analysis found that the role of mothers' negative emotion management differed between the two countries. In Medellin-Colombia, mothers' perceived self-efficacy in managing negative emotions mediated the effect of perceived neighborhood violence on positive parenting. In Rome-Italy, mothers' self-efficacy in managing negative emotions did not mediate this link. Colombian mothers' perceived self-efficacy regarding negative emotion management indirectly explained the adverse effect of

\footnotetext{
Luz Magnolia Tilano Vega (D) https://orcid.org/0000-0001-9709-7985

Paula Luengo Kanacri (iD https://orcid.org/0000-0001-7228-459X

Concetta Pastorelli (D) https://orcid.org/0000-0003-2837-7312

Laura Di Giunta (D) https://orcid.org/0000-0002-2578-8610

Carolina Lunetti (D) https://orcid.org/0000-0001-6590-0534

Liliana Uribe Tirado (iD https://orcid.org/0000-0002-3218-0187

Eriona Thartori (iD https://orcid.org/0000-0001-6576-0834

Jennifer Lansford (iD https://orcid.org/0000-0003-1956-4917
}

El artículo es parte de la tesis de la primera autora, titulada "Predictores individuales y contextuales de crianza: comparación Medellín-Colombia y Roma-Italia", para optar al grado de Doctor en Psicología de la Universidad de San Buenaventura, Medellín. Esta investigación ha sido financiada por el Eunice Kennedy Shriver National Institute of Child Health and Human Development (NICHD), grant RO1-HD054805, el Fogarty International Center, grant RO3-TW008141, y el Intramural Research Program del National Institutes of Health, NICHD.

La correspondencia relativa a esta artículo debe ser dirigida a Luz Magnolia Tilano Vega, Facultad de Psicología, Universidad de San Buenaventura, San Benito, Carrera 56C N 51-110 Centro, Medellín, Colombia. Email: luz.tilano@usbmed.edu.co 
perceived neighborhood violence on positive parenting. In mothers from Rome-Italy, no significant association was found between perceived self-efficacy in the management of negative emotions and positive parenting.

Keywords: positive parenting, parent-child relationship quality, emotional self-efficacy, neighborhood violence

La crianza positiva hace referencia a diversas estrategias utilizadas por los padres que favorecen el bienestar infantil. Es considerada fundamental en la promoción del desarrollo infantil y en la promoción del desarrollo cerebral y social de los niños (Pastorelli et al., 2016; von Suchodoletz et al., 2011; Whittle et al., 2014). Es por esto que se recomienda su promoción como estrategia eficaz en los programas de formación de padres (United Nations Office on Drugs and Crime [UNDOC], 2013). Desde una perspectiva bioecológica (Bronfenbrenner, 1979/1987), se sabe que la acción de los padres y sus estrategias educativas están anidadas en un contexto social más amplio, con el cual interactúan dinámicamente. Uno de los ambientes proximales más relevantes en relación a las prácticas familiares es el barrio o territorio inmediato donde la familia se desarrolla. En particular, algunas investigaciones han subrayado el rol que actualmente ejercen los vecindarios violentos o inseguros en las prácticas de crianza de las familias. Se hace, entonces, crucial relevar el efecto nocivo que la exposición a los altos niveles de violencia y criminalidad que atraviesa la vida de algunas ciudades pueda tener en la crianza y los factores individuales capaces de ayudar a mitigar tal efecto.

En particular, en acuerdo con el modelo de Belsky (1984), en el que la crianza es concebida como una práctica parental multideterminada por factores de distinta naturaleza, sea individuales (de los padres o de los hijos) o contextuales, el presente estudio tuvo como objetivo principal evidenciar si la percepción de violencia de barrio puede afectar la autoeficacia materna en la gestión de las emociones negativas y si esta, a su vez, puede predecir la crianza de los hijos, específicamente la calidad de la relación madre-hijo. Dicho de otro modo, la creencia de las madres de poder manejar sus emociones negativas aumenta en la medida que se percibe que el contexto en el que se vive es seguro (o poco violento) y esta capacidad de manejar adecuadamente las propias reacciones emocionales negativas, a su vez, aumenta la calidad de la relación madre-hijo. Este estudio responde a un importante llamado de los académicos a investigar el rol de las emociones de los padres en la crianza, en este caso, la función mediadora de la autoeficacia percibida en la gestión de emociones negativas materna en la relación entre la percepción de violencia de barrio y las prácticas de crianza positiva que no ha sido abordada en estudios anteriores. Tampoco el enfoque comparativo entre culturas distintas ha sido considerado. Colombia e Italia son dos países representativos de culturas e idiosincrasias disímiles y el actual enfoque permite establecer cuán universales pueden ser las relaciones entre los mecanismos propuestos y cuán representativos son de la explicación de la crianza positiva en contextos culturales diferentes.

\section{La Crianza Positiva}

Se conocen diversas estrategias de crianza positiva. Una de ellas es la calidad de la relación definida como el conjunto de comportamientos que demuestran amor, calidez y afecto a los niños y no evidencian abandono o indiferencia, sino involucramiento (Rohner et al., 2005). Rohner (2004) ha descrito un continuum entre la aceptación y el rechazo en la relación padres/hijos. Se ha visto que la asociación entre la aceptación-rechazo y la adaptación del niño son invariantes en todos los países, en la medida que todos los niños en los distintos contextos culturales requieren ser aceptados por sus padres (Putnick et al., 2015). No obstante esta relación sea universal, factores como el género (Oburu, 2011) o la pobreza (Lordelo et al., 2000) pueden afectar tal continuum, lo que hace necesario estudiar los factores que pueden explicar la crianza positiva en función del contexto cultural.

\section{Exposición a la Violencia en Colombia e Italia y su Relación con la Crianza Positiva}

Los resultados de investigaciones no son consistentes con respecto al uso de la crianza en presencia de ambientes violentos. Se plantea mayor uso de crianza punitiva en madres expuestas a altos niveles de violencia en la comunidad, incluso en diferentes culturas (Lansford \& Dodge, 2008). Otras evidencias muestran cómo la rudeza es utilizada como medida extrema para guardar y proteger a la juventud (Mason et al., 1996). En cuanto a la calidad de la relación madre-hijo y su asociación con la percepción de violencia de barrio, Pinderhughes et al. (2001) realizaron un estudio en Estados Unidos con padres europeos americanos y afroamericanos de zonas urbanas (Durham, Carolina del Norte; Nashville, Tennessee y Seattle, Washington) y rurales (ciudades pequeñas del centro de Pensilvania). En éste evidenciaron que la calidez de los padres y la disciplina apropiada 
presentan una relación inversa con la violencia de barrio, lo que podría indicar que, ante el estrés crónico de vivir en un barrio con mucha violencia y delincuencia, los padres son menos capaces de reunir la energía necesaria para ser cálidos, consistentes y no agresivos con sus hijos. Sin embargo, es necesario investigar de manera más profunda cuándo los comportamientos de los padres y menor calidez con sus hijos varían en función del contexto violento o de valores culturales generales.

\section{Autoeficacia Materna en la Gestión de las Emociones Negativas}

El concepto de autoeficacia desarrollado desde la perspectiva social cognitiva (Bandura, 2001) hace referencia a las creencias que las personas tienen sobre la capacidad de actuar para alcanzar determinados objetivos o hacerle frente a situaciones difíciles. Recientemente se han abordado diferentes creencias de autoeficacia asociadas con el dominio de la regulación emocional (Bandura et al., 2003). La autoeficacia emocional negativa tiene que ver con la capacidad percibida para gestionar la vida emocional propia, específicamente la capacidad para manejar estados emocionales negativos (Caprara \& Steca, 2006), es decir, la convicción de las personas en la propia capacidad de enfrentar adversidades o eventos frustrantes y de no ser vencidos por emociones como el enojo, la irritación, el desaliento y el desánimo (Bandura et al., 2003; Caprara et al., 2008).

La autoeficacia emocional de las madres, como dimensión individual que tiene un rol en la crianza, ha sido poco abordada; sin embargo, se ha visto que, frente a circunstancias difíciles o estresores relevantes, las personas que no modulan suficientemente sus emociones pueden externalizar sus sentimientos inapropiadamente en las relaciones familiares (Eisenberg et al., 2001).

Finalmente, algunos estudios han relacionado el contexto de barrio con la autorregulación emocional y la crianza. Edler Jr. et al. (1995), al analizar los efectos de la presión económica en el distrés emocional y el comportamiento parental de padres afroamericanos y europeos americanos, encontraron que en las familias con privaciones y fuerte estrés económico disminuye significativamente la eficacia parental. Otros estudios analizaron cómo la tensión financiera disminuye el bienestar emocional de los padres, lo que afecta negativamente su capacidad parental (McLoyd, 1998). Todavía no se conocen estudios que hayan examinado el efecto de la autoeficacia percibida por los padres para gestionar las emociones negativas en la crianza de los hijos.

\section{El Presente Estudio}

Si bien el estudio es parte de un proyecto internacional de investigación, el proyecto Parenting Across Cultures (PAC; Lansford et al., 2005), que involucra a nueve países, entre ellos Colombia e Italia, estos dos países fueron seleccionados por ser grupos culturales disímiles en dimensiones que pudieren afectar la crianza, la segmentación en estratos socioeconómicos, la orientación psicológica individualista y el nivel de violencia percibida.

Colombia ha sido considerado uno de los países con más peligrosidad percibida del mundo. En Sudamérica está incluido, junto con Brasil y Venezuela, entre los países con tasas persistentemente altas de homicidio, aunque ha experimentado una disminución dramática en la tasa de homicidios, desde más de 80 por 100.000 habitantes en 1991 a 25 en 2017, lo que se ha atribuido parcialmente a la intensificación de la acción del Estado contra el narcotráfico (UNDOC, 2019). El Instituto Nacional de Medicina Legal y Ciencias Forenses reveló que en 2018 los departamentos con las tasas de homicidios más altas fueron Arauca $(59,10)$, Valle del Cauca $(47,81)$, Putumayo $(46,53)$, Caquetá $(41,51)$ y Norte de Santander $(39,17)$, zonas en que el conflicto armado se ha recrudecido con la presencia de diferentes grupos violentos (Grupo Centro de Referencia Nacional sobre Violencia, 2018).

En el sur de Europa, las tasas de homicidios han disminuido de manera constante pasando de un 39\% a un $65 \%$ en las últimas décadas, específicamente de 2,3 por 100.000 habitantes en 1990 a 0,8 en 2017. En el caso de Italia, en 2017 con una tasa de 1,2, disminuyó alrededor de una cuarta parte de su tasa en 1990 (UNDOC, 2019). A pesar de que el crimen organizado (mafia) afecta a la población, principalmente en Sicilia, Campaña, Calabria y Puglia, en el 2018 el número de homicidios por cada 100.000 personas fue de 0,43 en el centro-norte y de 0,57 en el sur (Istituto Nazionale di Statistica, 2020).

Esta diversidad proporcionó la oportunidad de examinar una interacción de variables que no se había abordado en investigaciones anteriores. En base a la literatura antes expuesta, si bien no enteramente 
correspondiente a las dimensiones analizadas, pero cercanas a las mismas, se espera, en primer lugar, que la percepción de violencia de barrio se asocie inversamente con la calidad de la relación madre-hijo (Lansford \& Dodge, 2008). Además, se espera que esta asociación se explique indirectamente por la autoeficacia percibida por la madre en la gestión de las emociones negativas, la que está asociada inversamente con la percepción de violencia de barrio (Edler Jr. et al., 1995) y predice, a su vez, directamente la calidad de la relación madre-hijo (Barros et al., 2015). En segundo lugar, si las asociaciones son invariantes entre Colombia e Italia, las relaciones entre las características individuales y contextuales analizadas en este estudio con la crianza positiva utilizada por las madres serán compatibles entre ambas muestras. De acuerdo con el modelo de proceso de crianza propuesto por Belsky (1984), en el cual se plantea que la crianza está determinada por la personalidad de los padres, las características de los hijos y por factores contextuales que afectan la relación padre-hijo, como la red social de los padres. En este sentido, varios estudios (e.g., Furstenberg Jr. et al., 1993; Garbarino \& Kostelny, 1993) han demostrado que vivir en un barrio peligroso afecta la calidad de la relación entre padres e hijos y las prácticas de crianza de los padres. Específicamente, en comparación con los padres que viven en barrios más seguros, los padres que viven en barrios violentos y de alto riesgo tienden a ser menos cálidos y a usar una disciplina más dura como práctica de crianza. Con base en estos estudios previos, en este estudio se formuló la hipótesis de que el efecto negativo de vivir en un barrio percibido como peligroso sobre la crianza positiva puede ser mayor en Colombia, donde la tasa de delincuencia y violencia es mayor que en Italia. Hubo una inclinación a plantear la hipótesis de que las madres colombianas que tienen que enfrentar diariamente el crimen y la violencia experimentan un estrés crónico que compromete sus habilidades parentales y socava la calidad de la relación madre-hijo.

\section{Método}

\section{Participantes}

Se seleccionó una muestra de 434 madres e hijos (108 díadas de Medellín-Colombia y 109 díadas de Roma-Italia). Las pruebas $t$ de Student mostraron diferencias significativas entre las muestras de ambos países con mayores promedios en Roma-Italia para la edad de los niños, las madres y el nivel educativo de éstas. El rango de edad de los niños fue de 8 a 12 años $(M=9,73, D E=0,68$ y $M=10,51, D E=0,77$ en Colombia e Italia, respectivamente), $t(191)=7,403, p<0,001,95 \%$ IC $[0,569,0,982]$. La edad de las madres, entre 25 y 57 años $(M=37,95, D E=7,39$ y $M=42,63, D E=5,00$ en Colombia e Italia, respectivamente), $t(191)=5,122, p<0,001,95 \%$ IC $[2,880,6,488]$. La cantidad de años de educación de las madres (considerado un factor indicativo de estatus socioeconómico), Colombia $(M=10,64, D E=5,21)$ e Italia $(M=13,72, D E=4,00)$, $t(191)=4,582, p<0,001,95 \%$ IC $[1,754,4,406]$. La distribución de la muestra por género en ambos países fue de $45 \%$ y $53,5 \%$ de varones en Colombia e Italia, respectivamente.

La muestra en Colombia se seleccionó de manera proporcional al número de personas por estrato socioeconómico reportadas por la Dirección Nacional de Estadística y en Italia se seleccionó según el grupo social acorde a los datos del Istituto Nazionale di Statistica.

La selección de las escuelas en Colombia fue de tipo no probabilístico por conveniencia, seleccionando instituciones que tenían algún tipo de relación con el Consultorio Psicológico Popular de la Universidad de San Buenaventura. Se seleccionaron 11 escuelas públicas de los estratos 1, 2 y 3 (más bajos) y 12 escuelas privadas pertenecientes a los estratos 4, 5 y 6 (más altos). Solo tres de las escuelas contactadas (pertenecientes a los estratos 3, 4 y 6) no quisieron participar en la investigación. Completaron el estudio 108 familias, de las cuales 12 (11\%) son de estrato 1, 38 familias (35\%) de estrato 2, 25 familias (23\%) de estrato 3, 14 familias (13\%) de estrato 4, 11 familias (10\%) de estrato 5 y 8 familias (7\%) de estrato 6.

En Italia con un muestreo no probabilístico por conveniencia, se enviaron cartas de presentación de la investigación y formularios solicitando información de contacto a los padres de dos escuelas públicas de Roma. Para asegurar una cobertura de los diferentes estratos sociales, también se publicaron volantes en una parroquia, dos centros recreativos públicos y uno financiado con fondos privados en diferentes áreas metropolitanas. De las familias que respondieron a la solicitud de participar en la investigación, 109 completaron el estudio. Dado que en Italia no existe una tipología de estratos similar a la colombiana, se tuvo en cuenta que la muestra coincidiera con las estadísticas nacionales (Istituto Nazionale di Statistica, 2020) en cuanto a la situación socioeconómica, la ocupación y la educación en el momento en que se inició el estudio. En detalle, el 4\% de las madres informó que los ingresos familiares anuales eran de unos 5.000 euros, el 3\% de 5.000 a 10.000 euros, el $5,1 \%$ de 11.000 a 15.000 euros, el $28,3 \%$ de 16.000 a 29.000 euros, el $25,3 \%$ de 
30.000 a 40.000 euros, el $10,1 \%$ de 41.000 a 50.000 euros, el $6,1 \%$ de 51.000 a 60.000 euros, el $11 \%$ de 61.000 a 80.000 euros y el 7,1\% más de 81.000 euros. El 62\% de las madres informó que, en comparación con el año anterior, los ingresos familiares no cambiaron, el 4,1\% y el 17,3\% informó que los ingresos familiares se han reducido mucho y se han reducido un poco, respectivamente, mientras que el 18,3\% informó que los ingresos familiares han aumentado.

En ambos países solo participó un hijo de familias con presencia de ambos padres en el hogar. Se seleccionaron participantes que no tuvieran diagnósticos previos de trastornos o enfermedades médicas de gravedad.

\section{Instrumentos}

\section{Autoeficacia Percibida de Gestión de Emociones Negativas en la Madre}

Se utilizó la escala Emotional Self-Efficacy desarrollada por Caprara et al. (2008), que evalúa la capacidad percibida de las personas para manejar sus emociones positivas (5 ítems) y negativas (9 ítems). Las afirmaciones de este cuestionario están redactadas en términos de lo que la persona cree que puede hacer, es decir, cuánto se percibe capaz para enfrentar determinada acción. Los criterios incluyen: 1 (para nada capaz), 2 (poco capaz), 3 (medianamente capaz), 4 (muy capaz) y 5 (del todo capaz). El instrumento posee validez interna, es multidimensional y puede ser utilizado en países occidentales y no occidentales (Caprara et al., 2008; Gunzenhauser et al., 2013). Se evaluó la multidimensionalidad de la escala RESE desarrollada para el contexto italiano, explorando si los factores hipotéticos separados surgen para la autoeficacia de emociones positivas y negativas, así como si más de un factor surge por la dimensión postulada de la gestión de emociones negativas. También se probó la generalización de la estructura latente multidimensional de la escala en otra cultura occidental (Estados Unidos) y en un país más bien distante de Italia y los Estados Unidos en las condiciones de vida y las tradiciones (Bolivia) calculando las pruebas de invariancia primero por sexo en cada país y luego a través de las culturas. Por último, se examinó la validez convergente de las subescalas obtenidas y se realizaron retrotraducciones. El análisis exploratorio y confirmatorio apoyó la existencia de tres factores, uno para la autoeficacia percibida para expresar afecto positivo y dos para la autoeficacia percibida para gestionar emociones negativas: desánimo/angustia y para controlar la ira/irritación. Se aceptaron todos los ítems cargados por encima de 0,30 en sus respectivos factores y no en otros factores.

Para este estudio se utilizaron cuatro ítems correspondientes a las creencias de las madres de autoeficacia para gestionar emociones negativas frente al desánimo y la irritación, por ejemplo, "Cuán capaz se cree de evitar desanimarse frente a la adversidad" o "Cuán capaz se cree de no perder el control cuando tiene rabia". Se seleccionaron de acuerdo con los valores arrojados en los análisis de fiabilidad. Los valores del Alfa de Cronbach si el ítem es excluido fueron los siguientes para Medellín-Colombia y Roma-Italia respectivamente: Ítem 1 (0,784/0,710); Ítem 2 (0,795/0,490); Ítem 4 (0,771/0,545); Ítem 4 (0,761/0,515). Se calcularon las medias de cada subescala y las puntuaciones más altas reflejaron niveles más altos de autoeficacia percibida de gestión de emociones negativas en las madres.

El alfa de Cronbach de esta dimensión fue 0,82 para la muestra de Medellín-Colombia y 0,64 para RomaItalia. Siguiendo a Serbetar \& Sedlar (2016) por el bajo número de ítems este alfa se ha considerado adecuado, lo que muestra apropiada consistencia interna.

\section{Percepción de Violencia de Barrio}

La percepción de violencia del barrio se evaluó por madres e hijos, con el fin de evitar un reporte insuficiente de percepción de violencia desde la perspectiva de una sola persona, que pudiera subestimar el peligro al que están expuestos y su efecto (Skinner et al., 2014). Se incluyeron siete ítems de una escala diseñada para evaluar la peligrosidad del barrio desde la perspectiva de las personas, utilizada previamente por O'Neill et al. (2001). Las afirmaciones son referidas a la zona en la cual viven, por ejemplo, "Muchos muchachos en mi barrio se meten en problemas", "Hay mucha droga y pandillas en mi barrio", "Mi barrio es un lugar peligroso para vivir", "Siento miedo en mi barrio". La escala de respuesta de 5 puntos va de 1 (muy en desacuerdo) a 5 (muy de acuerdo). Se promediaron los ítems de madres e hijos, los puntajes más altos hicieron referencia a una percepción más alta de violencia de barrio. 
El alfa de Cronbach para la muestra de Medellín-Colombia fue 0,88 y para la de Roma-Italia, 0,77, mostrando adecuada consistencia interna.

\section{Crianza Positiva}

La crianza positiva se midió considerando la calidad de la relación madre-hijo. Para esto se utilizó la forma corta del cuestionario de Aceptación-Rechazo/Control para padres diseñada por Rohner (2005). Comprende 29 ítems, que evalúan las actitudes y sentimientos de aceptación y rechazo de las madres desde la percepción de los hijos. Posee cuatro subescalas: calidez/afecto, abandono/indiferencia, descuido/ hostilidad y rechazo. Para este estudio se derivó una sub-escala para medir calidez/indiferencia sacando el promedio de seis ítems cuyos valores fueron invertidos dado que estaban redactados de manera negativa en la subescala de negligencia, relativos a la indiferencia (por ejemplo, "Mi madre no presta atención a mí cuando pido ayuda" quedó como "Mi madre me presta atención cuando le pido ayuda"). Los niveles de respuesta fueron: 1 (casi nunca), 2 (una vez al mes), 3 (una vez a la semana) y 4 (todos los días). Se sumaron los ítems y una puntuación más alta corresponde a mayor calidad de la relación.

Este instrumento posee validez convergente y discriminante (Rohner, 2005). El instrumento ha sido utilizado en diferentes países, mostrando confiabilidad superior a 0,70 en todos los grupos evaluados (Khaleque \& Rohner, 2002). El alfa de Cronbach para Colombia fue de 0,61 y para Italia de 0,62.

Se aseguró la equivalencia de los instrumentos a nivel conceptual y lingüístico con un procedimiento de traducción y retrotraducción, llamado back-translation (Maxwell, 1996). Los traductores poseían tanto un español como un italiano fluido.

\section{Factores sociodemográficos}

Se llevó a cabo una encuesta inicial a las madres con diferentes variables de interés para el proyecto científico PAC del cual forma parte esta investigación. Este formulario de información familiar incluyó datos como estado civil, creencia religiosa, empleo, etnia, conformación familiar, estrato socioeconómico e ingresos anuales brutos. Para el presente análisis se incluyeron: la educación de las madres entendida como el número de años cursados y aprobados, y el género de los niños ( $0=$ masculino; $1=$ femenino).

\section{Procedimiento}

Se recibió aprobación del comité de bioética, como investigación con riesgo mínimo, de las universidades San Buenaventura de Medellín y Sapienza di Roma. En ambos países, tras la aprobación de los directores de las escuelas y líderes de los lugares recreativos y parroquias, se les informó a los padres sobre el estudio y se concertaron entrevistas con cada familia interesada. Tanto padres como hijos firmaron el consentimiento informado.

Los instrumentos se aplicaron entre febrero y marzo de 2013, por psicólogos profesionales previamente capacitados. Las aplicaciones se llevaron a cabo de forma individual a la madre y al hijo en habitaciones separadas en las casas u otros sitios como instalaciones de las universidades participantes, escuelas o lugares de eventos barriales. La duración promedio de todos los instrumentos del proyecto PAC fue de una hora para madres e hijos.

Al final de la aplicación de los instrumentos, las familias recibieron un bono proporcional al costo de vida en cada país, 50.000 pesos en Colombia y 50 euros en Italia.

\section{Análisis de Datos}

Se examinaron las estadísticas descriptivas y alfas de Cronbach para evaluar la consistencia interna de los instrumentos y las correlaciones bivariadas entre las variables del estudio antes de realizar el análisis multivariante. Todas las variables endógenas tenían una distribución normal (asimetría $<2,00$; curtosis $<7,00$ ). Con el fin de testear la relación planteada en la hipótesis sobre el papel mediador de la autoeficacia percibida para la gestión de emociones negativas entre el predictor contextual y la calidad de la relación madre-hijo, a modo de ofrecer mayor comprensión para estudios futuros en términos de su papel como factor protector de la crianza positiva, se llevó a cabo un análisis de mediación estadística (MacKinnon et al., 2007), considerando como variable dependiente la calidad de la relación madre-hijo, como variable mediadora la autoeficacia materna en la gestión de las emociones negativas y como variable independiente la percepción de violencia 
de barrio. Se utilizó como variable de control el género de los hijos. El ajuste del modelo se evaluó siguiendo los procedimientos estándar: $\chi^{2}$ coeficiente de probabilidad estadística, los índices de ajuste comparativo (CFI y TLI), y el error de la raíz cuadrada media de aproximación, RMSEA y el SRMR, con intervalos de confianza del 90 por ciento asociados. Debido a que el $\chi^{2}$ es sensible a la muestra grande, se calcularon otros índices de ajuste del modelo a los datos: el índice comparativo de Bentler-Bonett (CFI), el índice de Tucker Lewis (TLI), la raíz del error cuadrático medio de aproximación (RMSEA) y la raíz del residuo cuadrático medio estandarizado (SRMR). Se consideró CFI y TLI > 0,90, RMSEA < 0,08, y SRMR < 0,05 como indicativos de ajuste aceptable del modelo (Kline, 2011). Se utilizaron los límites asimétricos de confianza para la distribución del producto del efecto indirecto $(a b)$ indicados por MacKinnon et al. (2002). Los valores superiores e inferiores de los límites de confianza del 90\% (IC) se calcularon utilizando el programa PRODCLIN (Tofighi \& MacKinnon, 2011).

Además, dentro del enfoque de ecuaciones estructurales, se realizó una comparación multigrupos, utilizando Mplus 7.0 (2012-2016), para testear la invarianza del modelo a nivel de países. En el enfoque multigrupo, la equivalencia entre ambos países fue evaluada imponiendo restricciones de igualdad entre los parámetros del modelo. La plausibilidad de estas restricciones de igualdad se examinó con el test $\chi^{2}$, el que prueba si la diferencia entre los modelos anidados (es decir, la solución en la que todos los parámetros fueron restringidos a ser iguales y la solución de base sin restricciones) cambia significativamente. El modelo se comporta de manera invariante si las restricciones de igualdad no producen un aumento significativo de $\chi^{2}$.

\section{Resultados}

En la Tabla 1 pueden observarse los datos descriptivos de cada escala, según el país, y en la Tabla 2, las correlaciones entre las variables consideradas en este estudio. Tal como se presentan en la Tabla 1, los valores de alfa de Cronbach para la variable violencia de barrio muestran buena consistencia interna en ambos países, para la variable autoeficacia percibida para la gestión de emociones negativas revelan buena consistencia en Medellín-Colombia y adecuada en Roma-Italia y para la variable calidad de la relación madrehijo la consistencia interna fue considerada aceptable en Medellín-Colombia y Roma-Italia, teniendo en cuenta la longitud de la escala este alfa se ha considerado adecuado (Serbetar \& Sedlar, 2016).

Tabla 1

Índices Descriptivos y Consistencia Interna de cada Escala para Medellín-Colombia y Roma-Italia

\begin{tabular}{llcccc}
\hline $\begin{array}{c}\text { Ciudad- } \\
\text { país }\end{array}$ & \multicolumn{1}{c}{ Constructo } & $\begin{array}{c}\text { Número } \\
\text { de ítems }\end{array}$ & Media & $D E$ & $\begin{array}{c}\text { Alfa de } \\
\text { Cronbach }\end{array}$ \\
\hline Medellín- & Percepción de violencia de barrio & 7 & 0,64 & 0,60 & 0,881 \\
Colombia & Autoeficacia de emociones negativas & 4 & 3,47 & 0,77 & 0,824 \\
& Calidad de la relación madre-hijo & 6 & 3,63 & 0,47 & 0,606 \\
Roma- & Percepción de violencia de barrio & 7 & 0,75 & 0,39 & 0,768 \\
Italia & Autoeficacia de emociones negativas & 4 & 3,21 & 0,63 & 0,644 \\
& Calidad de la relación madre-hijo & 6 & 3,71 & 0,36 & 0,616 \\
\hline
\end{tabular}

Con referencia a los cut-off relativos a la interpretación de la fuerza de los coeficientes de correlación, siguiendo a Cohen (1988), en la Tabla 2 puede evidenciarse una correlación inversa de fuerza moderada entre la autoeficacia materna en la gestión de las emociones negativas y la percepción de violencia de barrio. Esto significa que al aumentar la percepción de violencia de barrio disminuye la autoeficacia materna en la gestión de las emociones negativas. En cuanto a la crianza positiva, se observa una correlación inversa de fuerza moderada entre la calidad de la relación madre-hijo y la percepción de violencia de barrio, lo que expresa que a mayor percepción de violencia de barrio las madres utilizan menos demostraciones de amor, calidez y afecto con sus hijos. La autoeficacia materna en la gestión de las emociones negativas no muestra relación con la calidad de la relación madre-hijo. 
Tabla 2

Correlaciones entre las Variables del Modelo

\begin{tabular}{lccc}
\hline \multicolumn{1}{c}{ Variable } & $\begin{array}{c}\text { Percepción de } \\
\text { violencia de barrio }\end{array}$ & $\begin{array}{c}\text { Autoeficacia de } \\
\text { emociones negativas }\end{array}$ & $\begin{array}{c}\text { Calidad de la } \\
\text { relación }\end{array}$ \\
\hline Percepción de violencia de barrio & - & & \\
Autoeficacia de emociones negativas & $-0,19^{*}$ & - & - \\
Calidad de la relación & $-0,36^{* *}$ & 0,13 & - \\
\hline
\end{tabular}

${ }^{*} p<0,05,{ }^{* *} p<0,01$.

De acuerdo al análisis multigrupo (Colombia e Italia), la diferencia de $\chi^{2}$, comparando el modelo restringido, $\chi^{2}(7, n=199)=13,922, p=0,052$, con el modelo sin restricciones, $\chi^{2}(2, n=199)=0,489, p=0,783$, fue significativa, $\left.\Delta \chi^{2}(5, n=199)=12,433, p=0,019\right)$, lo que sugiere que la igualdad de todos los parámetros no se mantiene en ambos países. Después de haber examinado los índices de modificación, se consideró que el parámetro de autoeficacia percibida en la gestión de las emociones negativas sobre la calidad de la relación podía comportarse de modo diferencial en ambos países. En este caso, la diferencia de $\chi^{2}, \Delta \chi^{2}(4, n=199)=2,77$, $p=0,597$, no siguió siendo significativa, sugiriendo la plausibilidad de un modelo parcialmente restringido e invariante, en el que, como se observa en la Figura 1, el peso de la variable autoeficacia percibida en la gestión de las emociones negativas sobre calidad de la relación es significativo para Colombia y no para Italia. El modelo final, parcialmente restringido, presenta buenos índices de adaptación, $\chi^{2}(6, n=199)=3,259, p=0,775$, $\mathrm{CFI}=1,00, \mathrm{TLI}=1,00, \mathrm{RMSEA}=0,00,90 \% \mathrm{IC}[0,00,0,08], \mathrm{SRMR}=0,058$.

Como se observa en la Figura 1, las relaciones entre la violencia de barrio, la calidad de la relación madrehijo y la autoeficacia percibida para la gestión de las emociones negativas son casi todas significativas en ambos países (salvo el ya mencionado parámetro diferencial de la relación entre autoeficacia en la gestión de las emociones negativas y calidad de la relación, que en el caso de Italia no es significativo). El efecto de mediación o efecto indirecto se muestra significativo, ya que el IC no incluye el 0 en el caso de Colombia, 95\% IC [-0,051, -0,002], a diferencia del caso de Italia, 95\% IC [-0,003, 0,045]. Estimando la relación directa en el mismo modelo, excluyendo la variable mediadora (autoeficacia en la gestión de las emociones) el coeficiente beta estandarizado de percepción de violencia de barrio sobre la calidad de la relación es de $0,28,(p<0,001)$ para Roma-Italia y de 0,41 $(p<0,001)$ para Medellín-Colombia. Se observa, por lo tanto, que, incluyendo la variable mediadora, la relación directa entre percepción de violencia de barrio y calidad de la relación madre-hijo disminuye 0,02 y 0,04, respectivamente. Es decir, en la muestra de familias colombianas, el efecto de la percepción de violencia de barrio sobre la calidad de la relación madre-hijo incide doblemente: pasa indirectamente por la presencia de la autoeficacia materna en la gestión de las emociones negativas, la que es más baja si la percepción de violencia de barrio aumenta, y la autoeficacia materna en la gestión de las emociones negativas predice significativamente la calidad de la relación madre-hijo. Con las madres italianas, no se evidencia una relación significativa entre la autoeficacia percibida para la gestión de emociones negativas con la crianza positiva.

\section{Discusión}

Este estudio avanza en la comprensión de una dimensión de la crianza positiva que es la calidad de la relación madre-hijo en asociación con características individuales de personalidad de la madre (la autoeficacia materna en la gestión de las emociones negativas) y características contextuales (exposición a violencia del barrio), comparando Italia y Colombia, como países representativos de prácticas culturales y parentales diversas. Tal como fue hipotetizado, en ambos países se evidenció una asociación inversa entre la percepción de violencia de barrio y la calidad de la relación madre-hijo, siendo más fuerte en MedellínColombia, respecto de Roma-Italia. En la comparación realizada entre países se evidenció que, más allá del género de los niños, en términos globales el modelo de relación entre la autoeficacia materna en la gestión de las emociones negativas y la calidad de la relación madre-hijo no se comporta igualmente en ambos países. La percepción de violencia de barrio en los dos países se asocia inversamente con la autoeficacia materna en la gestión de las emociones negativas; sin embargo, en el caso de Medellín-Colombia, a diferencia de RomaItalia, mientras más altos son los niveles de esta autoeficacia percibida de las madres mejor es la calidad de 
la relación madre-hijo. En este sentido, en Colombia la relación inversa del contexto (percepción de violencia de barrio) con la crianza positiva es explicada parcialmente por la presencia de la autoeficacia en la gestión de las emociones negativas de las madres.

\section{Figura 1}

Modelo de Mediación Multigrupo para Colombia e Italia

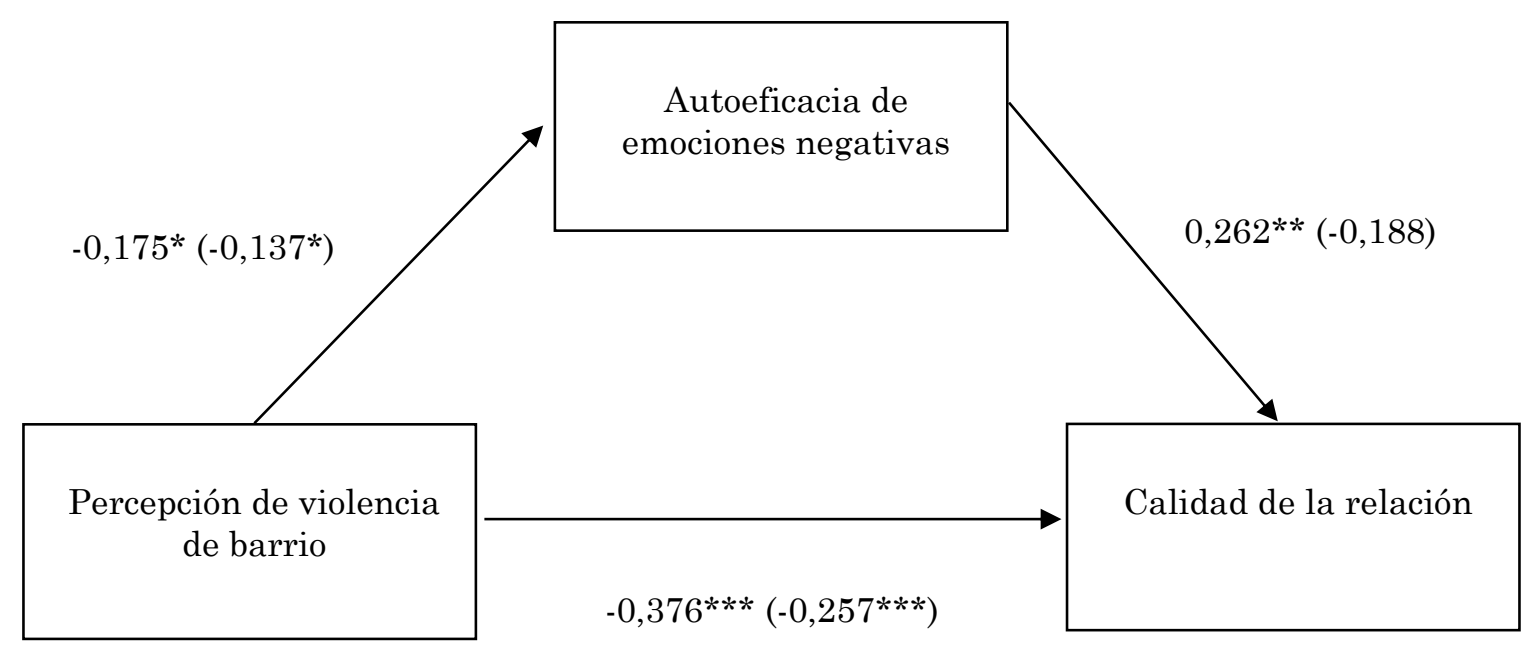

Nota. Se presentan valores de beta estandarizados. Los valores entre paréntesis representan a Italia. El efecto del género como covarianza no está dibujado en la figura, ya que no fue significativo en todos los casos. ${ }^{*} p<0,05 ;{ }^{* *} p<0,01 ; * * * p<0,001$.

Respecto a la relación directa entre la percepción de violencia de barrio con la calidad de la relación madre-hijo, estos resultados son coherentes con lo esperado en esta investigación y lo observado en investigaciones anteriores que asocian la desventaja socioeconómica con la crianza menos positiva y otros aspectos negativos del espectro de conductas parentales, que se expresan en la crianza menos sensible y receptiva, la disciplina más severa y la menor orientación a los niños (McLoyd et al., 2006).

Una asociación fuerte en Colombia entre la percepción de la violencia de barrio sobre la calidad de la relación madre-hijo es coherente con lo hallado por Pinderhughes et al. (2001) sobre niveles más bajos de calidez de los padres y mayores niveles de crianza dura en familias expuestas a barrios violentos. Sin embargo, la convicción de las madres colombianas de saberse capaces de manejar sus emociones de rabia o frustración explica parcialmente por qué a mayor percepción de estar en un contexto inseguro o violento se observa una menor calidad de la relación madre-hijo. Es decir, la convicción de las madres en Colombia de poder gestionar sus emociones aumentaría en la medida que se percibe que el contexto en el que se vive es poco violento y esta capacidad de manejar adecuadamente las propias reacciones emocionales de rabia o frustración, a su vez, aumentaría la calidad del vínculo con sus hijos. Este dato, que evidencia un factor protector típico de un ambiente cultural como Colombia, ofrece interesantes aspectos informativos para intervenciones que pretendan mitigar los efectos deletéreos que condiciones de barrios con altos índices de violencia pueden tener en el desarrollo de los niños. Aun en ambientes y barrios altamente marcados por la violencia, madres más convencidas de poder gestionar sus emociones negativas pueden ser más competentes emocionalmente, lo que les permitiría expresar afecto y atención a sus hijos, cualidades propias de la calidad de la relación parental, necesarias en una crianza positiva.

A pesar de los planteamientos de investigaciones anteriores sobre la influencia del género de los hijos como factor que pudiese afectar la aceptación de los padres y la responsividad con sus hijos (Oburu, 2011), en este estudio no se observó que el género de los hijos, utilizado como variable de control, tuviese algún efecto en las variables involucradas.

Es preciso reconocer algunas limitaciones de esta investigación. Se tomaron solo mediciones en las diversas variables en un solo momento en el tiempo, por lo cual no se analizó un período más largo que 
permitiera dar cuenta de los efectos directos e indirectos en un análisis longitudinal, lo que daría más fuerza a la relación causal entre las variables. Además de la utilización del género de los niños como variable de control, sería recomendable controlar otras variables como el nivel socioeconómico y la composición familiar. En esta investigación se tuvo en cuenta la valoración del cumplimiento de los supuestos básicos para el análisis de consistencia interna con alfa de Cronbach de los diferentes constructos analizados. Se consideran adecuados los alfas superiores a 0,60 de las muestras estudiadas para la variable calidad de la relación teniendo en cuenta el número bajo de ítems. Consideramos por lo tanto que los ítems de la escala miden un mismo constructo y están altamente correlacionados entre sí; sin embargo, una recomendación para futuras investigaciones sería considerar procedimientos que incrementen la fiabilidad y validez del constructo, una alternativa metodológica podría ser el uso de la omega de McDonald o si es del caso incluir un mayor número de ítems para los análisis factoriales. Por último, las muestras extraídas de cada país no fueron representativas para Medellín-Colombia ni Roma-Italia, por lo tanto, los resultados no se deben generalizar o ser interpretados como reflejo de toda una cultura.

No obstante estas limitaciones, los presentes hallazgos apoyan el valor de ciertas dimensiones individuales o psicológicas sobre condiciones estructurales adversas, ofreciendo espacio para una intervención psicosocial centrada en las personas y en los grupos.

\section{Referencias}

Bandura, A. (2001). Social cognitive theory: An agentic perspective. Annual Review of Psychology, 52, 1-26. https://doi.org/10.1146/annurev.psych.52.1.1

Bandura, A., Caprara, G. V., Barbaranelli, C., Gerbino, M. \& Pastorelli, C. (2003). Role of affective self-regulatory efficacy in diverse spheres of psychosocial functioning. Child Development, 74(3), 769-782. https://doi.org/10.1111/1467-8624.00567

Barros, L., Goes, A. R. \& Pereira, A. I. (2015). Parental self-regulation, emotional regulation and temperament: Implications for intervention. Estudos de Psicologia, 32(2), 295-306. https://doi.org/10.1590/0103-166X2015000200013

Belsky, J. (1984). The determinants of parenting: A process model. Child Development, 55(1), 83-96. https://doi.org/10.2307/1129836

Bronfenbrenner, U. (1987). La ecología del desarrollo humano: experimentos en entornos naturales y diseñados (A. Devoto, Trad.). Paidós Ibérica. (Obra original publicada en 1979).

Caprara, G. V., Di Giunta, L., Eisenberg, N., Gerbino, M., Pastorelli, C. \& Tramontano, C. (2008). Assessing regulatory emotional selfefficacy in three countries. Psychological Assessment, 20(3), 227-237. https://doi.org/10.1037/1040-3590.20.3.227

Caprara, G. V. \& Steca, P. (2006). The contribution of self-regulatory efficacy beliefs in managing affect and family relationships to positive thinking and hedonic balance. Journal of Social \& Clinical Psychology, 25(6), 603-627. https://doi.org/10.1521/jscp.2006.25.6.603

Cohen, J. (1988). Statistical power analysis for the behavioral Sciences: Second edition. Lawrence Erlabaum.

Edler, Jr., G. H., Eccles, J. S., Ardelt, M. \& Lord, S. (1995). Inner-city parents under economic pressure: Perspectives on the strategies of parenting. Journal of Marriage and Family, 57(3), 771-784. https://doi.org/10.2307/353931

Eisenberg, N., Cumberland, A., Spinrad, T. L., Fabes, R. A, Shepard, S. A., Reiser, M., Murphy, B. C., Losoya, S. H \& Guthrie, I. K. (2001). The relations of regulation and emotionality to children's externalizing and internalizing problem behavior. Child Development, 72(4), 1112-1134. https://doi.org10.1111/1467-8624.00337

Furstenberg, Jr., F. F., Belzer, A., Davis, C., Levine, J. A., Morrow, K. \& Washington, M. (1993). How families manage risk and opportunity in dangerous neighborhoods. En W. J. Wilson (Ed.), Sociology and the public agenda (pp. 231-258). SAGE.

Garbarino, J. \& Kostelny, K. (1993). Neighborhood and community influences on parenting. En T. Luster \& L. Okagaki (Eds.). Parenting: An ecological perspective (pp. 203-226). Lawrence Erlbaum.

Grupo Centro de Referencia Nacional sobre Violencia. (2018). Forensis 2018: datos para la vida [Conjunto de datos]. República de Colombia, Instituto Nacional de Medicina Legal y Ciencias Forenses, Subdirección de Servicios Forenses. https://www.medicinalegal.gov.co/documents/20143/386932/Forensis+2018.pdf/be4816a4-3da3-1ff0-2779-e7b5e3962d60h

Gunzenhauser, C., Heikamp, T., Gerbino, M., Alessandri, G., von Suchodoletz, A., Di Giunta, L, Caprara, G. V. \& Trommsdorff, G. (2013). Self-efficacy in regulating positive and negative emotions: A validation study in Germany. European Journal of Psychological Assessment, 29(3), 197-204. https://doi.org/10.1027/1015-5759/a000151

Istituto Nazionale di Statistica. (2020). Le vittime di omicidio. Anno 2018. https://www.istat.it/it/files//2020/03/Report-Le-vittime-di-omicidio.pdf

Khaleque, A. \& Rohner, R. P. (2002). Reliability of measures assessing the pancultural association between perceived parental acceptance-rejection and psychological adjustment: A meta-analysis of cross-cultural and intracultural studies. Journal of CrossCultural Psychology, 33(1), 87-99. https://doi.org/10.1177/0022022102033001006

Kline, R. B. (2011). Principles and practices of structural equation modeling: Third edition. Guilford Press.

Lansford, J. E., Chang, L., Dodge, K. A., Malone, P. S., Oburu, P., Palmérus, K., Bacchini, D., Pastorelli, C., Bombi, A. S., Zelli, A., Tapanya, S., Chaudhary, N., Deater-Deckard, K., Manke, B. \& Quinn, N. (2005). Physical discipline and children's adjustment: Cultural normativeness as a moderator. Child Development, 76(6), 1234-1246. https://doi.org/10.1111/j.1467-8624.2005.00847.x

Lansford, J. E. \& Dodge, K. A. (2008). Cultural norms for adult corporal punishment of children and societal rates of endorsement and use of violence. Parenting, 8(3), 257-270. https://doi.org/10.1080/15295190802204843

Lordelo, E. R., Fonseca, A. L. \& Lamêgo, M. V. B. A. (2000). Responsividade do ambiente de desenvolvemento: crenças e práticas como sistema cultural de criação de filhos [Respuesta del entorno de desarrollo: creencias y prácticas como sistema cultural de crianza]. Psicologia: Reflexão e Crítica, 13(1), 73-80. https://doi.org/10.1590/S0102-79722000000100009

MacKinnon, D. P., Fritz, M. S., Williams, J. \& Lockwood, C. M. (2007). Distribution of the product confidence limits for the indirect effect: Program PRODCLIN. Behavior Research Methods, 39(3), 384-389. https://doi.org/10.3758/BF03193007

MacKinnon, D. P., Lockwood, C. M., Hoffman, J. M. \& West, S. G. (2002). A comparison of methods to test mediation and other intervening variable effects. Psychological Methods, 7(1), 83-104. https://doi.org/10.1037/1082-989X.7.1.83 
Mason, C. A., Cauce, A. M., Gonzales, N. \& Hiraga, Y. (1996). Neither too sweet nor too sour: Problem peers, maternal control, and problem behavior in African American adolescents. Child Development, 67(5), 2115-2130. https://doi.org/10.1111/j.1467-8624.1996.tb01847.x

Maxwell, B. (1996). Translation and cultural adaptation of the survey instruments. En M. O. Martin \& D. L. Kelly (Eds.), Third international mathematics and science study (TIMSS) technical report. Volume I: Design and development (pp. 1-10). Boston College.

McLoyd, V. C. (1998). Socioeconomic disadvantage and child development. American Psychologist, 53(2), 185-204. https://doi.org/10.1037/0003-066X.53.2.185

McLoyd, V. C., Aikens, N. L. \& Burton, L. M. (2006). Childhood poverty, policy, and practice. En N. Eisenberg (Eds.), Handbook of child psychology. Volume 4: Child psychology in practice. Sixth edition (pp. 700-775). John Wiley \& Sons. https://doi.org/10.1002/9780470147658.chpsy0418

Oburu, P. O. (2011). Attributions and attitudes of mothers and fathers in Kenya. Parenting, 11(2-3), 152-162. https://doi.org/10.1080/15295192.2011.585561

O'Neil, R., Parke, R. D. \& McDowell, D. J. (2001). Objective and subjective features of children's neighborhoods: Relations to parental regulatory strategies and children's social competence. Journal of Applied Developmental Psychology, 22(2), 135155. https://doi.org/10.1016/S0193-3973(01)00073-9

Pastorelli, C., Lansford, J. E., Luengo Kanacri, B. P., Malone, P. S., Di Giunta, L., Bacchini, D., Bombi, A. S., Zelli, A., Miranda, M. C., Bornstein, M. H., Tapanya,S., Uribe, L. M., Peña L., Al-Hassan, S. M., Chang, L., Deater-Deckard, K., Dodge, K. A., Oburu, P., Skinner, A. T. \& Sorbring, E. (2016). Positive parenting and children's prosocial behavior in eight countries. Journal of Child Psychology and Psychiatry, 57(7), 824-834. https://doi.org/10.1111/jcpp.12477

Pinderhughes, E. E., Nix, R., Foster, E. M., Jones, D. \& The Conduct Problems Prevention Research Group. (2001). Parenting in context: Impact of neighborhood poverty, residential stability, public services, social networks, and danger on parental behaviors. Journal of Marriage and Family, 63(4), 941-953. https://doi.org/10.1111/j.1741-3737.2001.00941.x

Putnick, D. L., Bornstein, M. H., Lansford, J. E., Malone, P. S., Pastorelli, C., Skinner, A. T., Sorbring, E., Tapanya, S., Uribe, L. M., Zelli, A., Peña, L., Al-Hassan, S. M., Bacchini, D. Bombi, A. S., Chang, L., Deater-Deckard, K., Di Giunta, L., Dodge, K. A., \& Oburu, P. (2015). Perceived mother and father acceptance-rejection predict four unique aspects of child adjustment across nine countries. Journal of Child Psychology and Psychiatry, 56(8), 923-932. https://doi.org/10.1111/jcpp.12366

Rohner, R. P. (2004). The parental "acceptance-rejection syndrome": Universal correlates of perceived rejection. American Psychologist, 59(8), 830-840. https://doi.org/10.1037/0003-066X.59.8.830

Rohner, R. P. (2005). Parental acceptance-rejection/control questionnaire (PARQ/Control): Test manual. En R. P. Rohner \& A. Khaleque (Eds.), Handbook for the study of parental acceptance and rejection (4a ed., pp. 137-186). University of Connecticut.

Rohner, R. P., Khaleque, A. \& Cournoyer, D. E. (2005). Parental acceptance-rejection theory, methods, evidence, and implications. En R. P. Rohner \& A. Khaleque (Eds.), Handbook for the study of parental acceptance and rejection (4a ed., pp. 1-35). University of Connecticut.

Serbetar, I. \& Sedlar, I. (2016). Assessing reliability of a multi-dimensional scale by coefficient alpha. Revija za Elementarno Izobraževanje, 9(1/2), 189-196. https://journals.um.si/index.php/education/article/view/391

Skinner, A. T., Bacchini, D., Lansford, J. E., Godwin, J. W., Sorbring, E., Tapanya, S., Uribe, L. M., Zelli, A., Peña, L., Al-Hassan, S. M., Bombi, A. S., Bornstein, M. H., Chang, L. Deater-Deckard, K., Di Giunta, L., Dodge, K. A., Malone, P. S., Miranda, M. C., Oburu, P. \& Pastorelli, C. (2014). Neighborhood danger, parental monitoring, harsh parenting, and child aggression in nine countries. Societies, 4(1), 45-67. https://doi.org/10.3390/soc4010045

Tofighi, D. \& MacKinnon, D. P. (2011). RMediation: An R package for mediation analysis confidence intervals. Behavior Research Methods, 43(3), 692-700. https://doi.org/10.3758/s13428-011-0076-x

United Nations Office on Drugs and Crime. (2013). World drug report 2013. United Nations. http://www.webcitation.org/6u10yim34

United Nations Office on Drugs and Crime. (2019). World drug report 2019. United Nations. https://igarape.org.br/wpcontent/uploads/2019/08/Booklet_3.pdf

von Suchodoletz, A., Trommsdorff, G. \& Heikamp, T. (2011). Linking maternal warmth and responsiveness to children's self-regulation. Social Development, 20(3), 486-503. https://doi.org/10.1111/j.1467-9507.2010.00588.x

Whittle, S., Simmons, J. G., Dennison, M., Vijayakumar, N., Schwartz, O., Yap, M. B. H., Sheeber, L. B. \& Allen, N. B. (2014). Positive parenting predicts the development of adolescent brain structure: A longitudinal study. Developmental Cognitive Neuroscience, 8 , 7-17. https://doi.org/10.1016/j.den.2013.10.006

Fecha de recepción: Julio de 2019.

Fecha de aceptación: Octubre de 2020. 